\title{
Unconventional gene regulation in the CNS
}

\author{
Dysregulation of protein translation and RNA processing mechanisms in the brain can result in subtle, but widespread, \\ neurological disorders. A collection of Review and Perspective articles in this issue of Nature Neuroscience highlights \\ some of the more unconventional mechanisms of post-transcriptional modifications in the CNS.
}

$\mathrm{T}$ he central dogma of molecular biology as articulated by Francis Crick $^{1}$ proposed a straight-forward mechanism of transcribing information from DNA to protein-DNA is replicated, RNA is transcribed and protein translation ensues. Over time, biologists have uncovered many divergent mechanisms that add to the complexity of how genes are read out. Many such mechanisms of post-transcriptional regulation can be found in the brain, where different cell types and subregions utilize unique forms of post-transcriptional control to accommodate specific CNS developmental patterns and functions. In this issue of Nature Neuroscience, we present a collection of Reviews that highlight some of the recent advances in our understanding of unconventional gene regulation mechanisms in the brain, in the context of both health and disease.

The fidelity of information from DNA to RNA is maintained when the latter is transcribed from the former. However, RNA can also differ in its informational content from its parent DNA strand because the RNA sequence can be edited to provide a divergent nucleic acid and/or amino acid code from its parent DNA. On page 1518, Billy Li and George Church discuss the mechanism by which a specific RNA base can be edited after transcription. In particular, they examine adenosine-to-inosine RNA editing mediated by the enzyme adenosine deaminase and summarize the systematic approaches that have recently been taken to profile at a genome level and across tissues which RNAs can undergo such changes. The authors also discuss the possibility of RNA editing as a means to drive evolutionary divergence in brain structure and function across different phyla.

Although RNA editing can provide more diverse information coding of genetic material, the brain also needs to maintain information fidelity at the level of DNA because coding error can be deleterious. Cellular stress and natural breakage can damage the genetic material encoded in DNA, and most cell types in the body utilize DNA repair mechanisms that are based on replicative correction. However, given that neurons are post-mitotic and do not readily undergo cellular selfrenewal, they require a repair mechanism that does not depend on DNA replication. On page 1523, Peter McKinnon reviews the molecular mechanisms by which the nervous system overcomes this unique challenge to maintain genomic stability and repair its DNA, not only during the natural course of development, but also following natural experience, cellular insults, stress, aging and disease. In his review, the author discusses the activation of the genome surveillance pathway during brain development that either signals a stop to cell proliferation or initiates apoptosis to put a brake on neural development. The review also discusses what ensues when DNA breaks go uncorrected and the disorders that stem from the failure to maintain genome stability.
Unlike DNA, which can self-renew, proteins translated from RNA must be constantly replenished as protein levels change due to active degradation and passive decay. So-called de novo protein synthesis is vital for maintaining the shape and function of neurons, and neurodevelopmental diseases such as fragile X syndrome (FXS) are often associated with protein translation defects. In their review, Eric Klann and Jennifer Darnell discuss the latest progress in our understanding of the biology of fragile X mental retardation protein and its function as a RNA binding protein and a translational regulator. They also discuss the potential therapeutic framework for combating FXS given these translational defects. In a separate review, Mauro Costa-Mattioli and Lisa Monteggia summarize the recent advances in the target of rapamycin (mTOR) pathway function in the brain and how such mechanisms may be involved in neurodevelopmental and neuropsychiatric diseases, including autism spectrum disorders. Given that mTOR acts as a signaling node that can integrate extracellular molecular information, in part by regulating the initial steps of intracellular protein translation, the review critically examines the concept of mTOR signal transduction acting as a translational mediator that can affect long-term memory formation and other cognitive processes.

Finally, in their review on page 1544, Chunghun Lim and Ravi Allada highlight the complexity of post-transcriptional regulation of clock genes to mediate cellular timekeeping. Eukaryotic circadian timekeeping mechanisms utilize an elaborate non-operonic transcription feedback loop network in which one gene product can shut off the transcription of another to achieve oscillatory 24-h cycles. Lim and Allada discuss the recent discovery of transcription termination of clock regulators by RNA binding proteins, circadian defects caused by dysfunctional proteins in mRNA alternative splicing pathways, and various microRNAs that regulate the synchronization of peripheral clocks and master neural circadian pacemakers to control circadian output.

In the last decade, we have learned that gene regulation mechanisms are far more complex than we had imagined. Neurons are faced with additional unique challenges, with subtle changes distinguishing one neuron from its neighbors. New regulatory mechanisms are bound to emerge as research effort progresses, and it is likely that our understanding of the mechanisms of neuronal physiology and disease will hinge on our appreciation and understanding of the many different levels of regulation that can affect the most basic of functions-transcription from DNA to RNA to protein. We hope that these reviews give our readers a glimpse of the tantalizing complexity of this process.

1. Crick, F. Nature 227, 561-563 (1970). 